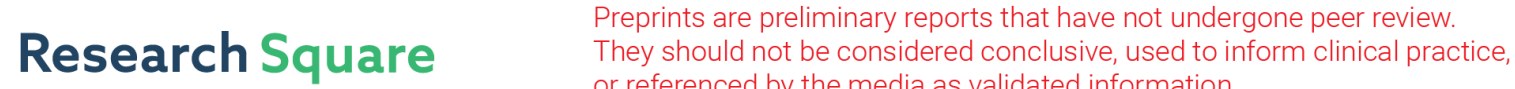 or referenced by the media as validated information. \\ Title Spironolactone for chronic central serous chorioretinopathy - a pilot study
}

\section{Xiaoli Yu}

The first people`s hospital of Nantong

Juanjuan Tang

Nanjing University of Chinese Medicine

Yu Song

The first people's hospital of Nantong

\section{Xin Cao}

The first people`s hospital of Nantong

\section{Tianwei Qian}

Shanghai general hospital

\section{Xinxin Li}

Shanghai general hospital

Suqin Yu ( $\sim$ sq-yu@163.com )

\section{Research article}

Keywords: chronic central serous chorioretinopathy, spironolactone, a pilot study

Posted Date: July 25th, 2019

DOI: https://doi.org/10.21203/rs.2.11867/v1

License: (c) (1) This work is licensed under a Creative Commons Attribution 4.0 International License.

Read Full License 


\section{Abstract}

AIM To estimate the efficacy of oral spironolactone in patients with chronic central serous chorioretinopathy (CSC). METHODS This was a pilot study consisting of patients who were diagnosed with chronic central serous chorioretinopathy for at least a 6-month duration. This study included 75 eyes of 74 patients who were treated with spironolactone ( $20 \mathrm{mg}$ orally, twice daily) for 8 weeks. The best corrected visual acuity (BCVA), subretinal fluid (SRF), central macular thickness (CMT) and subfoveal choroidal thickness (SFCT) were each measured at baseline, 4 weeks and 8 weeks. RESULTS In our study, 75 eyes of 74 patients ( 49 men and 25 women) were included. The mean BCVA was $0.39 \pm 0.25$ at baseline, $0.29 \pm 0.19$ at 4 weeks and $0.23 \pm 0.19$ at 8 weeks. The BCVA at 4 weeks and 8 weeks was significantly improved compared to the baseline BCVA $(p=0.000)$. The mean SRF and the mean CMT decreased significantly at 4 weeks and 8 weeks $(p=0.000)$. The mean SFCT decreased slightly at 4 weeks and 8 weeks but without significance compared to the baseline $(p=0.654)$. CONCLUSION Spironolactone can competitively bind to mineralocorticoid receptors (MRs), reduce glucocorticoid binding or reduce endogenous activation of MR. It is possible to improve the visual acuity and safety of patients with chronic CSC by reducing the high permeability of the choroid membrane and by promoting the absorption of the subretinal fluid.

\section{Background}

Central serous chorioretinopathy (CSC), which is characterized by subretinal fluid accumulation and retinal pigment epithelium (RPE) disruption, is self-limiting in most cases. However, CSC can be recurrent in elderly patients and can lead to severe, irreversible reduction of visual acuity. The precise pathogenesis and treatment of CSC are not fully understood. The abnormal choroidal vascular permeability, with a subsequent increase in hydrostatic pressure and disturbance of the overlying RPE in CSC, has been previously demonstrated ${ }^{1}$. Strikingly, an inappropriate activation of the mineralocorticoid receptor, which is found in the choroidal vasculature (endothelial and smooth muscle cells), has also been recently suggested to underlie the pathogenesis of $\mathrm{CSC}^{2}$. The features of CSC in an animal model have demonstrated optimal reversibility during treatment with the MR antagonist eplerenone ${ }^{2}$. This result was also proven in a small case series of chronic CSC patients ${ }^{3-4}$.

In recent reports, CSC patients who were treated with mineralocorticoid antagonists (spironolactone and eplerenone) achieved the same high rates of antiandrogen effects ${ }^{5}$, even when spironolactone was administered in a lower and therefore well tolerable dose, which can then lead to a lower rate of common side effects. In this study, we treated Chinese patients who were diagnosed as having chronic CSC with spironolactone.

\section{Ethical Approval}

This study was conducted in accordance with the Declaration of Helsinki and was approved by the ethics committee of Shanghai General Hospital. Informed consent was obtained from each patient. All patients 
underwent a thorough ophthalmic examination and agreed to receive trabeculectomy to reduce IOP.

\section{Subjects And Methods}

In our study, consecutive patients with chronic CSC between October 2014 and March 2018 at the Department of Ophthalmology of the Shanghai General Hospital were included. The inclusion criteria were as follows: (1) patients aged more than 18 years, (2) patients who had chronic CSC (duration $>6$ months) or recurrent chronic CSC and (3) patients who had FFA that revealed multiple RPE disruption. Persistent detachment of the RPE and/or the occurrence of subretinal fluid, as observed by optical coherence tomography (OCT), were also included as inclusion criteria. The exclusion criteria were (1) patients who had acute CSC; (2) patients who had the presence of choroidal neovascularization or other macular disorders; (3) patients who had eyes with a history of previous treatment, including laser photocoagulation (LP), photodynamic therapy (PDT) or intravitreal anti-vascular endothelial growth factor (anti-VEGF) injections; (4) patients who had cases of pregnancy, a history of steroid use and systemic diseases, such as endogenous hypercortisolism and diabetes; and (5) patients who had contraindications against spironolactone (severe liver or kidney disease, or serum potassium levels $>5.5$ $\mathrm{mmol} / \mathrm{L})$.

All of the patients were treated with spironolactone ( $20 \mathrm{mg}$ orally, twice daily) for 8 weeks. A complete ophthalmic evaluation, including best-corrected visual acuity (BCVA), slit-lamp biomicroscopy, fundus photography, fundus autofluorescence, raster scans of the macula using the enhanced depth imaging OCT (EDI-OCT) and FFA, was performed at baseline, 4 weeks and 8 weeks. The alterations in subretinal fluid, central macular thickness and subfoveal choroidal thickness measured via EDI-OCT were compared and analyzed among the baseline, 4-week and 8-week time points.

\section{Statistical Analysis}

Statistical analyses were performed by using SPSS 19.0 for Windows (SPSS 19.0 Inc., IBM Company, Chicago, IL, USA). Data are expressed as the median and range. P-Values were calculated with the Wilcoxon-test. A p-value $<0.05$ was significant.

\section{Results}

Seventy-five eyes of 74 patients (49 men and 25 women) were included in our study. The mean age was 49.7 years (median: 50.5 years; standard deviation (SD): +/-9.6).

The BCVAs (in logarithm of minimal angle resolution units) at baseline, 4 weeks and 8 weeks after spironolactone treatment are shown in Table 1 and Figure 1. The mean BCVAs were $0.39 \pm 0.25$ at baseline, $0.29 \pm 0.19$ at 4 weeks and $0.23 \pm 0.19$ at 8 weeks. Both of the BCVAs at 4 weeks and 8 weeks were significantly improved compared with the baseline BCVA $(p=0.000)$. 
The mean SRF decreased from $218 \mu \mathrm{m}$ (range: $36-662 \mu \mathrm{m})$ (baseline) to $75 \mu \mathrm{m}$ (range: 0-550 $\mu \mathrm{m})(8$ weeks) (difference: $143 \mu \mathrm{m}$ ) (Table 1 and Figure 2). A complete regression of the SRF at 8 weeks was achieved in 29.3\% (22/75) of the patients; however, 6 patients exhibited an increase in the SRF. The overall reduction of the SRF from baseline to visits 2 and 3 was statistically significant $(p=0.000)$.

For the EDI-OCT evaluation, the CMT decreased significantly from $397 \pm 132 \mu \mathrm{m}$ at baseline to $310 \pm 103$ $\mu \mathrm{m}$ and $254 \pm 103 \mu \mathrm{m}$ at 4 weeks and 8 weeks, respectively (Table 1 and Figure 3 ). The differences between baseline and 4 weeks and 8 weeks were statistically significant $(p=0.000)$. Fifteen patients had no significant changes in the CMT at 8 weeks, with these patients exhibiting a CMT decrease of $<20 \%$.

The mean SFCT measurements at baseline and during the follow-up period are summarized in Table 1 and Figure 4. The mean SFCT was $309.88 \pm 73.08 \mu \mathrm{m}$ at baseline, and the mean SFCT decreased slightly to $305.69 \pm 69.81 \mu \mathrm{m}$ and $300.29 \pm 68.60 \mu \mathrm{m}$ at 4 weeks and 8 weeks, respectively. However, the change from baseline was not significant $(p=0.654)$.

Two examples of the EDI-OCT courses of patients who received spironolactone treatment are provided in Figure 5. The fundus photography and fundus autofluorescence for a 43-year-old male patient are shown in Figure 6.

\section{Discussion}

The occurrence, development and recurrence of CSC may be related to changes in steroid hormone levels caused by mental tension, stress and other factors. Glucocorticoids are closely related to the incidence of CSC6. Studies have reported that there is a simultaneous expression of glucocorticoid receptors (GR), mineralocorticoid receptors (MR) and type 2 hydroxysteroid dehydrogenase (hsd-2) in retinal and choroid tissues, thus demonstrating that the retina and the choroid are novel targets of mineralocorticoids. In rats, the mineralocorticoid receptor specifically binds to aldosterone or high doses of glucocorticoids, and both aldosterone and glucocorticoids can activate receptors and cause the dilation and leakage of the choroid vessels, whereas MR antagonists can reverse these effects2, 7. Therefore, competitive antagonists of the MR are likely to be involved in the pathogenesis of CSC in the choroid. Zhao M. et al.2 have identified that aldosterone upregulates the endothelial vasodilatory potassium channel $\mathrm{KCa} 2.3$, which is an endothelial, hyperpolarizing, calcium-dependent channel that is involved in vasorelaxation. This endothelial hyperpolarizing factor pathway is one of the well-known vasorelaxation mechanisms that also occur in large vessels upon aldosterone activation.

Mineralocorticoid receptor antagonism reversed the upregulation and activation of this channel-induced vasodilation in the choroid. Interestingly, $\mathrm{KCa} 2.3$ is not expressed in retinal vessels but is only expressed in choroid vessels, thus explaining that steroids do not induce vasodilation of the retinal vessels upon MR activation. MR antagonists primarily involve spironolactone and eplerenone, which are primarily used in the treatment of hypertension, aldosteronism and congestive heart failure. The most common side effects are hyperkalemia and low blood pressure. In view of the self-limitation of CSC, MR antagonists are currently used to treat chronic CSC patients. A one-year pilot study by Ghadiali, Quraish MD5 
demonstrated that mineralocorticoid antagonists may improve best-corrected visual acuity and decrease subretinal fluid in patients with central serous chorioretinopathy, but these antagonists do not affect the choroidal or macular thicknesses. Nevertheless, the latest results of Sun X, et al.8 suggest that oral spironolactone is a promising treatment for acute CSC because it provides a faster absorption of SRF. Therefore, 75 eyes of 74 patients with chronic CSC were included in this study, and the efficacy

and safety were observed after 8 weeks of treatment with oral spironolactone.

The results of this study demonstrated that SRF was significantly absorbed in chronic CSC patients after 4 weeks of oral spironolactone treatment compared with the baseline SRF, that the best-corrected visual acuity was obviously improved after 4 weeks of treatment compared with the baseline measurement, and that the central macular thickness also exhibited visual reduction on at the end of the 4 weeks of treatment. However, there was no significant difference in the subfoveal choroidal thickness after 4 weeks of oral spironolactone treatment compared with the baseline measurement. At 8 weeks of treatment, the SRF of patients was further absorbed, and both the BCVA and CMT were significantly improved compared with the measurements at previous time points, while the SFCT exhibited no significant difference. No obvious side effects were observed during medication.

A subsequent prospective pilot study by Bousquet et al.3 described 13 patients with CSC who were treated with eplerenone. In their study, visual acuity, central macular thickness and subretinal fluid parameters were measured, and the results showed that eplerenone treatment was associated with a significant reduction in central macular thickness and subretinal fluid level as well as an improvement in visual acuity.

Kapoor KG9 created a retrospective chart review of 32 CSC patients who were treated with spironolactone or eplerenone (50 mg p.o. bid.) or observation, and it was observed that both MR antagonists demonstrated statistically significant visual acuity improvement and subretinal fluid reduction at 1, 2 and 3 months compared to the baseline $(p<0.05)$. A total of $58.3 \%$ of patients had a complete resolution of the subretinal fluid at 2 months of MR antagonist treatment, compared to $12.5 \%$ of patients who were under observation $(p<0.05)$. There was no difference in the efficacy between eplerenone and spironolactone treatments. Spironolactone treatment exhibited increased side effects (8/12 patients, $75 \%)$ compared to eplerenone treatment $(3 / 12$ patients, $25 \% ; p<0.05)$. There is also a study from Herold TR10 that reported that 18 patients with chronic CSC who were treated with $25 \mathrm{mg}$ spironolactone twice daily for 12 weeks exhibited decreases in SRF and CMT as well as improvements in BCVA. These results are similar to our results. However, these research subjects were all foreigners, and our subjects are Chinese. Through our study, we observed that spironolactone treatment is also effective for Chinese people. Additionally, the dose used in our study was $20 \mathrm{mg}$ twice daily, whereas the other study used a dosage of $25 \mathrm{mg}$ twice daily 10, which indicates that the low dose is also effective. However, the OCT parameters for subfoveal choroidal thickness did not show significant changes with treatment, although, as previously reported, patients with exudative CSC and subretinal fluids did exhibit significant anatomical improvements. We inferred that perhaps because of the signal loss before the scleral- 
choroidal edge in some cases, it was difficult to establish clear data on the possible differences in the subfoveal choroidal thickness in intractable chronic CSC patients at baseline and during the follow-up period.

By reviewing the studies of recent years 3,8 4, 10, we observed an interesting result: the absorption of SRF was more obvious after the Chinese studies used MR antagonists than when the European studies used MR antagonists. Sun X, et al.8 demonstrated that complete resolution of SRF was achieved in $55.6 \%$ $(10 / 18)$ of patients, and our research demonstrated that complete resolution was achieved in $29.3 \%$ (22/75) of patients, where the patients were Chinese. Herold TR10 reported that the complete regression of subretinal fluid was documented in $25 \%$ of the study eyes, and Singh RP4 demonstrated that six eyes (35.3\%) within the study demonstrated a complete resolution of SRF upon completion of the treatments. Meanwhile, Bousquet E3 reported that a complete reabsorption of SRF was achieved in 3/12 patients $(25 \%)$, with those patients being European. Additionally, our study had a larger sample of patients than the previous studies. Although the causes have not yet been thoroughly studied, the differences may be due to changes in hormone levels in stress and the environments; therefore, more clinical studies of multicenter big data are necessary. The limitation of our study was that the follow-up period only consisted of 2 months, which was too short to assess the long-term safety and efficacy of MR antagonism for the treatment of intractable chronic CSC and the recurrence rates. Larger, prospective, randomized controlled clinical trials should be further investigated, with longer follow-up periods.

\section{Declarations}

Authors Xiaoli Yu and Suqin Yu conceived the study. Authors Juanjuan Tang and Xiaoli Yu wrote the draft of the manuscript. Author Juanjuan Tang and Xin Cao provided the illustrations. Authors Yu Song, Tianwei Qian and Xinxin Li selected the statistical methods and performed the data analysis.

Foundation: The study was supported by the Natural Science Foundation of Jiangsu Province (Grant No. BK20161044), the Natural Science Foundation of Nantong (Grant No.MS22015085).

Conflicts of Interest: Xiaoli Yu, None; Juanjuan Tang, None; Yu Song, None; Xin Cao, None; Tianwei Qian, None; Xinxin Li, None, Suqin Yu, None

\section{References}

1. Spaide, R. F.; Hall, L.; Haas, A.; Campeas, L.; Yannuzzi, L. A.; Fisher, Y. L.; Guyer, D. R.; Slakter, J. S.; Sorenson, J. A.; Orlock, D. A., Indocyanine green videoangiography of older patients with central serous chorioretinopathy. Retina 1996, 16 (3), 203-13.

2. Zhao, M.; Celerier, I.; Bousquet, E.; Jeanny, J. C.; Jonet, L.; Savoldelli, M.; Offret, O.; Curan, A.; Farman, N.; Jaisser, F.; Behar-Cohen, F., Mineralocorticoid receptor is involved in rat and human ocular chorioretinopathy. J Clin Invest 2012, 122 (7), 2672-9. 
3. Bousquet, E.; Beydoun, T.; Zhao, M.; Hassan, L.; Offret, O.; Behar-Cohen, F., Mineralocorticoid receptor antagonism in the treatment of chronic central serous chorioretinopathy: a pilot study. Retina 2013, 33 (10), 2096-102.

4. Singh, R. P.; Sears, J. E.; Bedi, R.; Schachat, A. P.; Ehlers, J. P.; Kaiser, P. K., Oral eplerenone for the management of chronic central serous chorioretinopathy. Int J Ophthalmol 2015, 8 (2), 310-4.

5. Ghadiali, Q.; Jung, J. J.; Yu, S.; Patel, S. N.; Yannuzzi, L. A., Central Serous Chorioretinopathy Treated with Mineralocorticoid Antagonists: A One-Year Pilot Study. Retina 2016, 36 (3), 611-8.

6. Haimovici, R.; Koh, S.; Gagnon, D. R.; Lehrfeld, T.; Wellik, S.; Central Serous Chorioretinopathy CaseControl Study, G., Risk factors for central serous chorioretinopathy: a case-control study. Ophthalmology 2004, 111 (2), 244-9.

7. Zhao, M.; Valamanesh, F.; Celerier, I.; Savoldelli, M.; Jonet, L.; Jeanny, J. C.; Jaisser, F.; Farman, N.; Behar-Cohen, F., The neuroretina is a novel mineralocorticoid target: aldosterone up-regulates ion and water channels in Muller glial cells. FASEB J 2010, 24 (9), 3405-15.

8. Sun, X.; Shuai, Y.; Fang, W.; Li, J.; Ge, W.; Yuan, S.; Liu, Q., Spironolactone versus observation in the treatment of acute central serous chorioretinopathy. Br J Ophthalmol 2018, 102 (8), 1060-1065.

9. Kapoor, K. G.; Wagner, A. L., Mineralocorticoid Antagonists in the Treatment of Central Serous Chorioretinopathy: A Comparative Analysis. Ophthalmic Res 2016, 56 (1), 17-22.

10. Herold, T. R.; Prause, K.; Wolf, A.; Mayer, W. J.; Ulbig, M. W., Spironolactone in the treatment of central serous chorioretinopathy - a case series. Graefes Arch Clin Exp Ophthalmol 2014, 252 (12), 1985-91.

\section{Table}

Due to technical limitations, table 1 is only available as a download in the supplemental files section

\section{Figures}




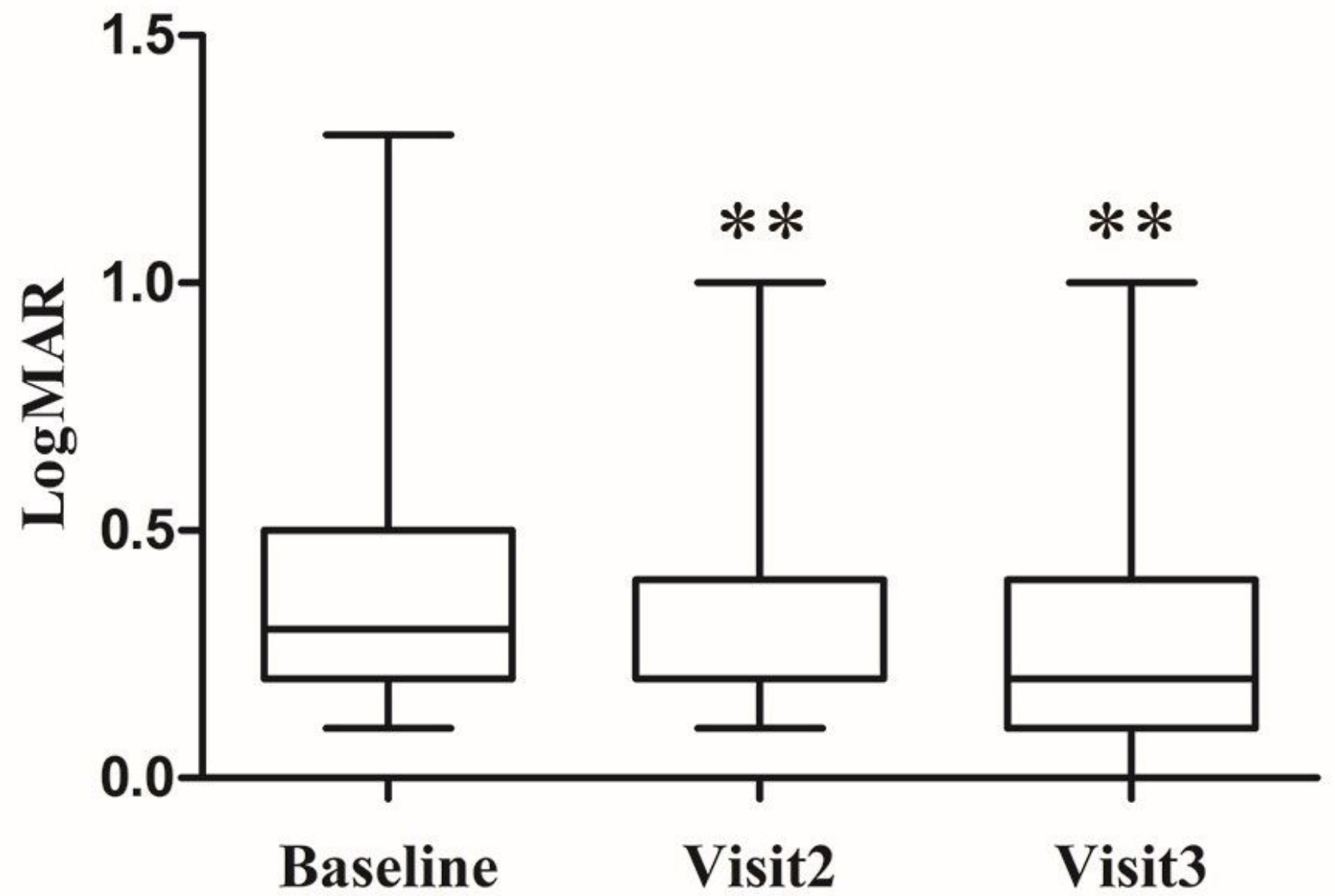

Figure 1

shows the BCVA (in logMAR) at baseline, 4 weeks and 8 weeks. ${ }^{*} \mathrm{P}<0.01$; Compared with baseline. 


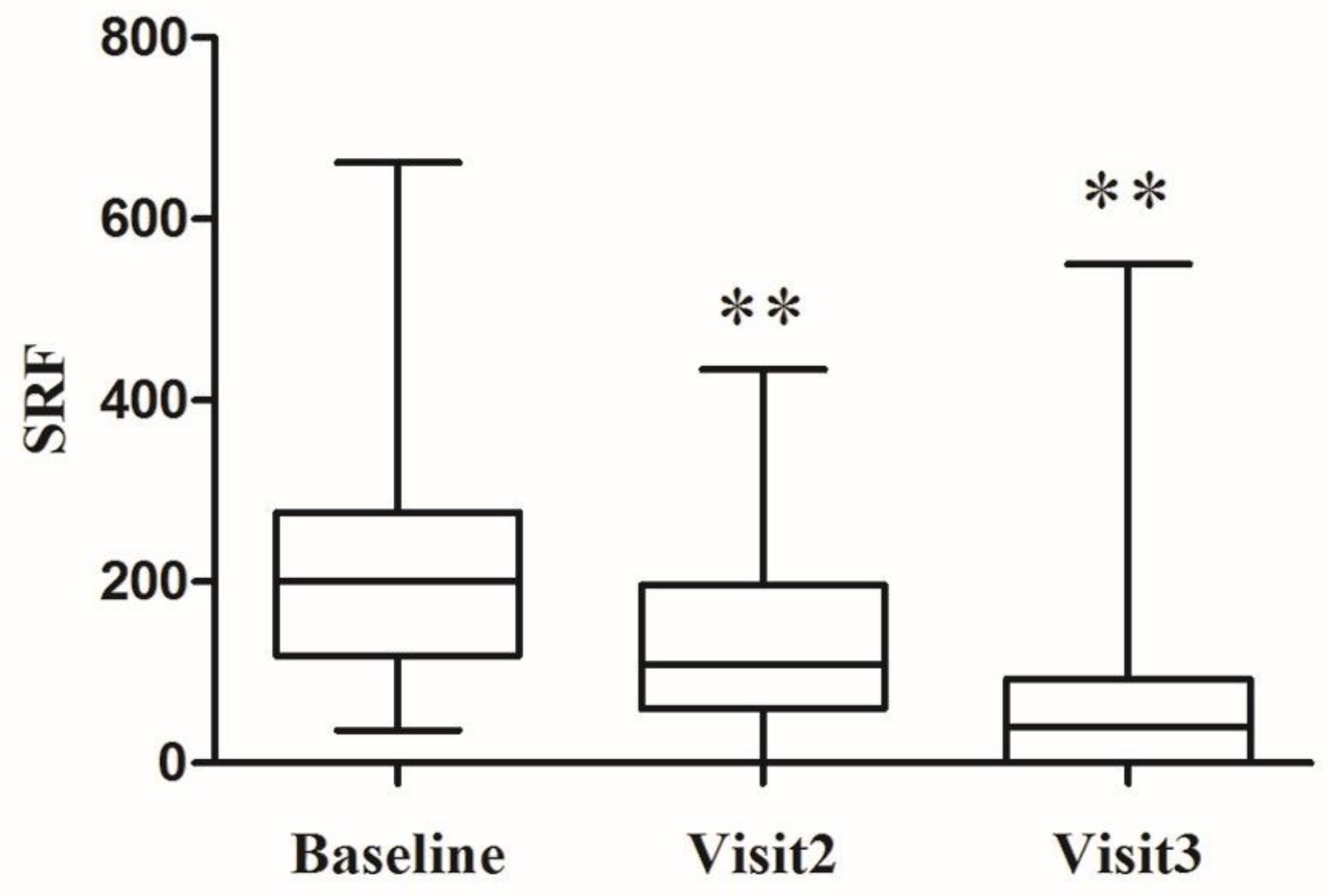

Figure 2

shows the SRF (in $\mu \mathrm{m}$ ) at baseline, 4 weeks and 8 weeks. ${ }^{\star *} \mathrm{P}<0.01$; Compared with baseline. 


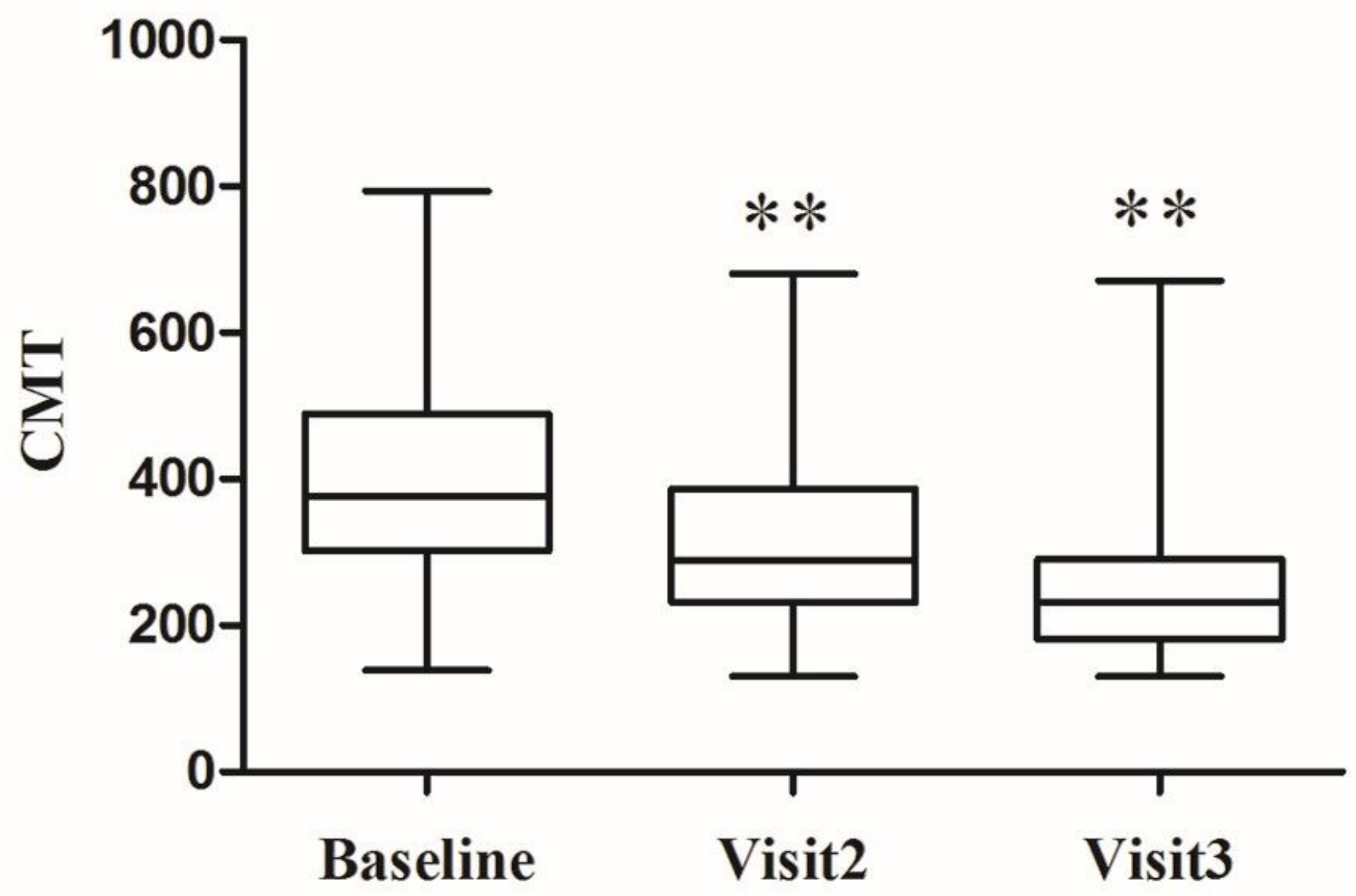

Figure 3

shows the CMT (in $\mu \mathrm{m}$ ) at baseline, 4 weeks and 8 weeks. ${ }^{* *} \mathrm{P}<0.01$; Compared with baseline. 


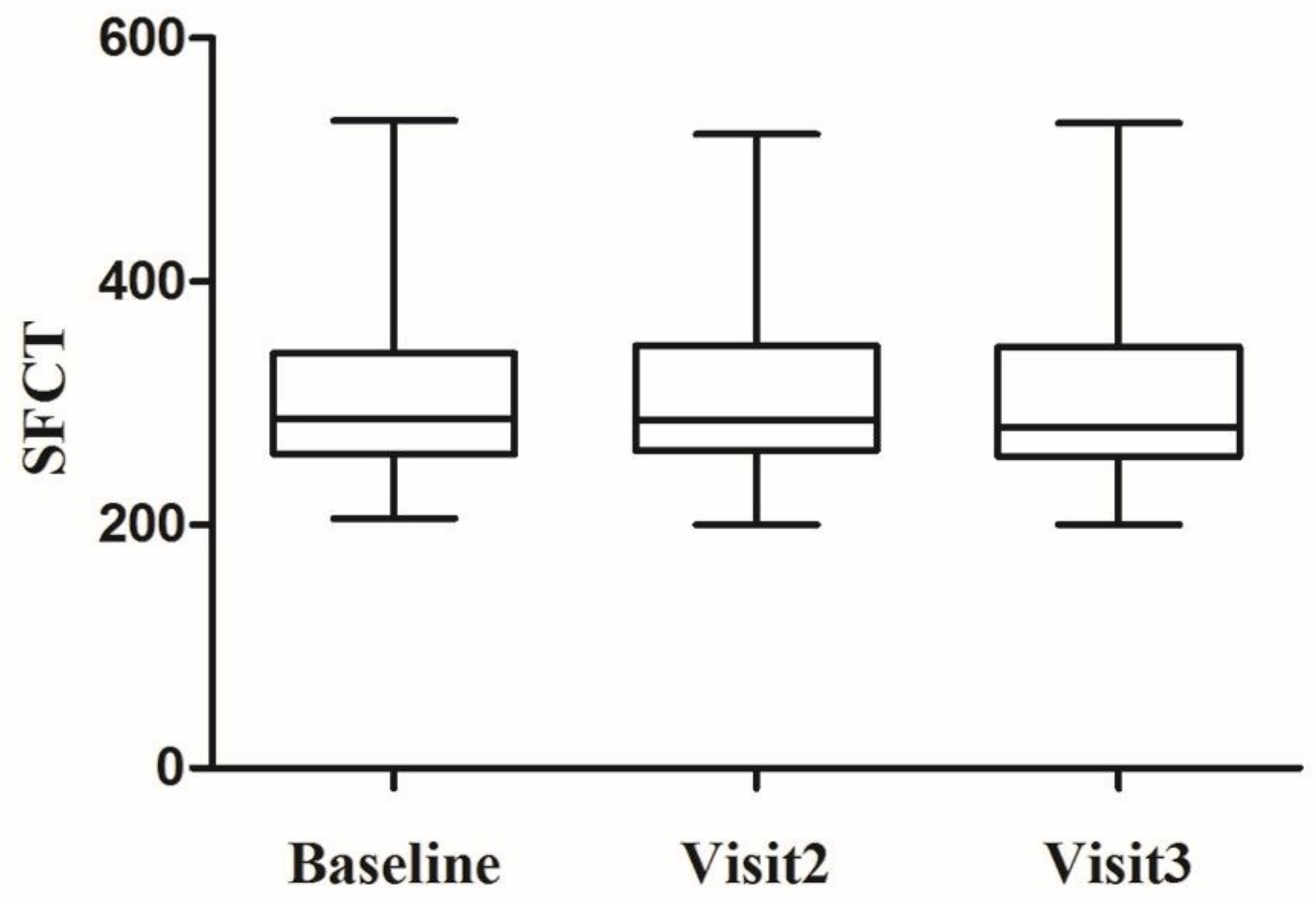

Figure 4

shows the SFCT (in $\mu \mathrm{m}$ ) at baseline, 4 weeks and 8 weeks. 


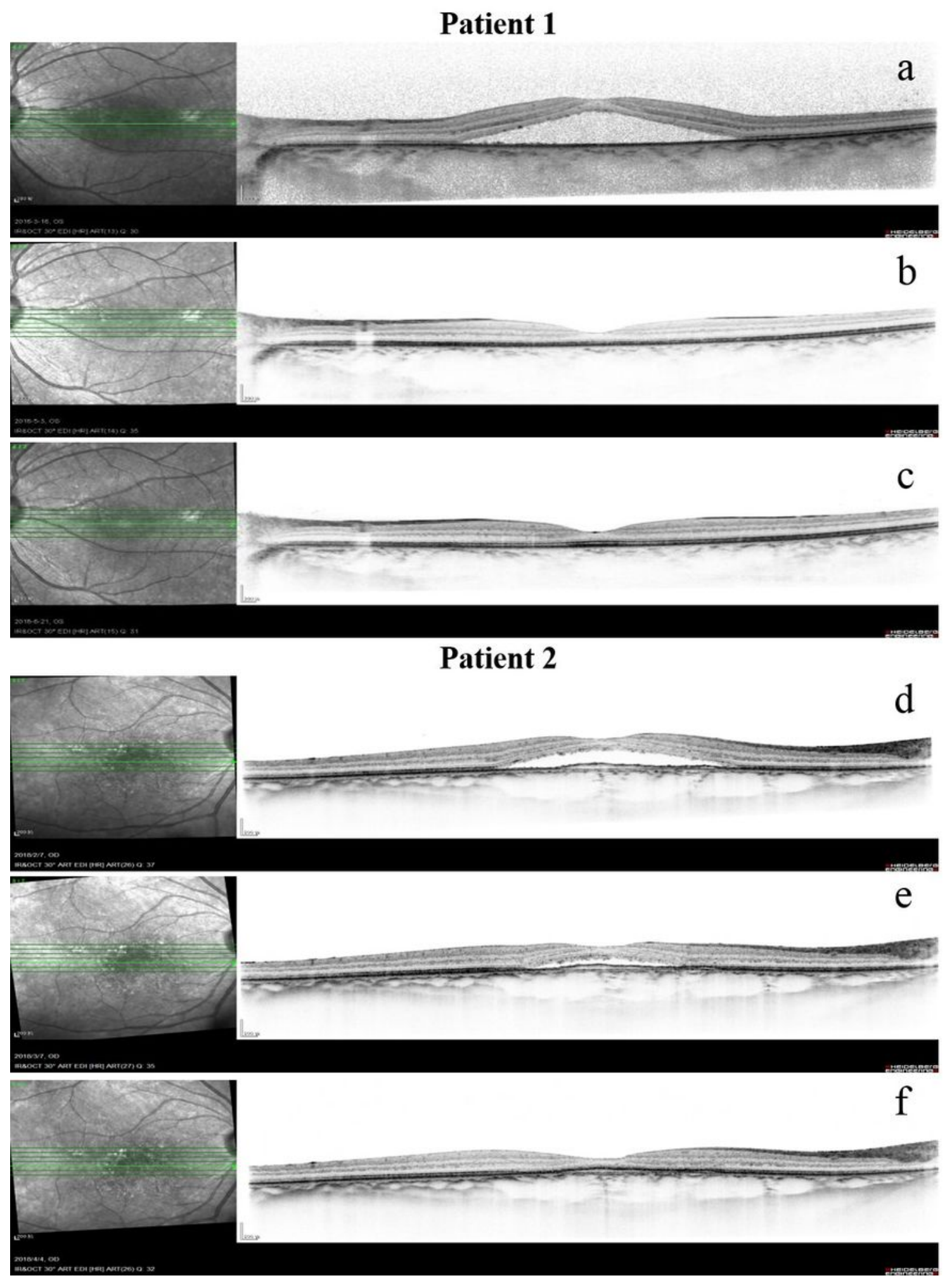

Figure 5

shows the enhanced depth imaging optical coherence tomography (EDI-OCT) pictures of two patients. a-c Baseline, 4 weeks and 8 weeks of a 30-year-old female patient with CSC. The BCVA increased from 0.3 logMAR (baseline) to 0.1 logMAR ( 8 weeks). $d$-f Baseline, 4 weeks and 8 weeks of a 54 -year-old male patient with CSC. The BCVA was $0.4 \log M A R$ at baseline and $0.1 \log$ MAR at 8 weeks. 


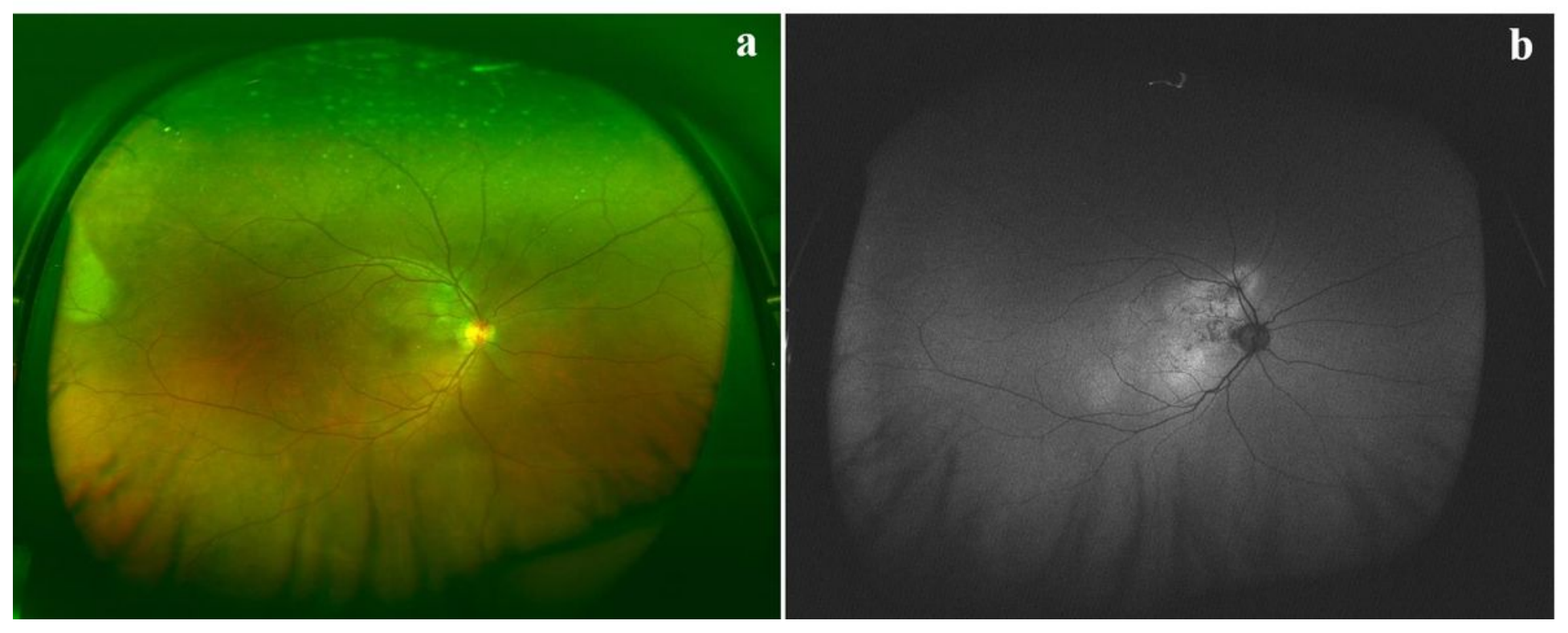

\section{Figure 6}

shows the fundus photography and fundus autofluorescence for a 43-year-old male patient. a fundus photography, b fundus autofluorescence.

\section{Supplementary Files}

This is a list of supplementary files associated with this preprint. Click to download.

- supplement1.tif 